DR-4

\title{
AN EFFICIENT METHOD FOR THE SYNTHESIS OF DIHYDROPYRIMIDINES USING BRØNSTED ACIDIC IONIC LIQUID: A SOLVENT AND HEATING FREE REACTION
}

\author{
Satyajit Samanta, ${ }^{1}$ Rana Chatterjee, ${ }^{1}$ Sougata Santra, ${ }^{2}$ Adinath Majee ${ }^{1}$ \\ ${ }^{1}$ Department of Chemistry; Visva-Bharati (A Central University), Santiniketan 731235, India. \\ ${ }^{2}$ Ural Federal University, 19 Mira St., Yekaterinburg-620002, Russian Federation. \\ E-mail: satyasamanta527@gmail.com
}

\begin{abstract}
Dihydropyrimidinones occupy a considerable position in the dominion of natural and synthetic organic chemistry, due to their various therapeutic and pharmacological properties, such as antiviral, antibacterial, antihypertensive and antitumor effects. ${ }^{1}$ Dihydropyrimidinones scaffold rose as mainstay of several drugs which are used as calcium channel blockers. ${ }^{2}$ Recently, isolated marine alkaloids have been connected with dihydropyrimidine-5-carboxylate core and found to be potent to HIV-gp-120 CD4 inhibitors. ${ }^{3}$ Years back, Biginelli reported the most simple and straight forward procedure for the synthesis of DHPMs. ${ }^{4}$ However, From bingeing, many methods have been developed for the synthesis of DHPMs and its derivative using various types of catalytic reagents. ${ }^{5}$ Considering the synthetic importance of Dihydropyrimidinones we developed an efficient and greener method for the synthesis of these compounds using Brønsted acidic ionic liquid (BAILs) \{1-Butane sulfonic acid3-methylimidazolium tosylate, [BSMIM]OTs $\}$ under solvent free condition and at room temperature (Scheme 1).
\end{abstract}

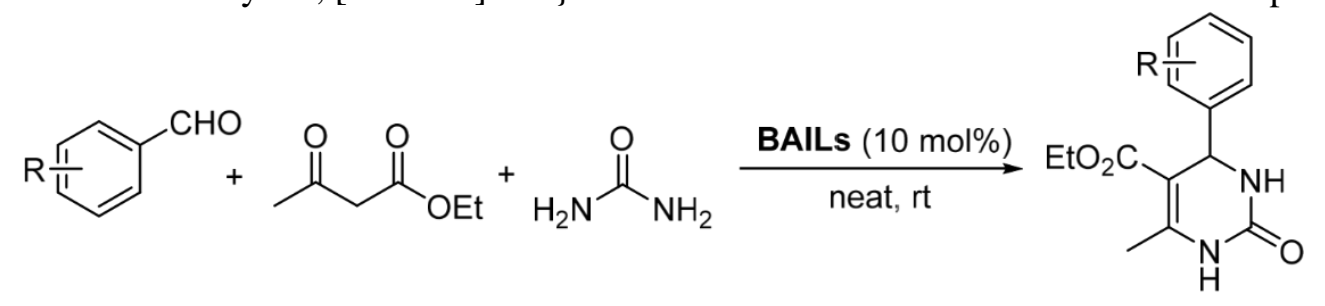

Scheme 1. Synthesis of Dihydropyrimidinones using BAILs

\section{References}

1. 100 years of the Biginelli dihydropyrimidine synthesis / O. Kappe // Tetrahedron. - 1993. - Vol. 49. - P. 6937-6963.

2. Dihydropyrimidine calcium channel blockers. 4. Basic 3-substituted-4-aryl-1,4dihydropyrimidine-5-carboxylic acid esters. Potent antihypertensive agents / G. C. Rovnyak, K. S. Atwal, A. Hedberg, S. D. Kimball, S. Moreland, J. Z. Gougoutas, B. C. OReilly, M. F. Malley // J. Med. Chem. - 1992. -Vol. 35. - P. 3254.

3. Biomimetic synthesis of (.+-.)-crambines A, B, C1, and C2. Revision of the structure of crambines B and C1 / B. B. Snider, Z. Shi // J. Org. Chem. - 1993. - Vol. 58 - P. 3828. 4. Biginelli Reaction / P. Biginelli // Ber. Dtsch. Chem. Ges. - 1893. - Vol. 26. - P. 447.

4. Lanthanide Triflate Catalyzed Biginelli Reaction. One-Pot Synthesis of Dihydropyrimidinones under Solvent-Free Conditions / Y. Ma, C. Qian, L. Wang, M. Yang // J. Org. Chem. - 2000, - Vol. 65. - P. 3864. 